\title{
Localized Synaptic Potentiation is Necessary and Sufficient for Context Fear Memory
}

Leonardo M. Cardozo ${ }^{1,2,3,4 \bowtie}$, Blythe C. Dillingham ${ }^{2,3,5}$, Andre F. de Sousa ${ }^{2,3,5}$, Westley Dang, ${ }^{2,3,5}$, Nicholas Job ${ }^{2,3}$, Eun J. Yoo ${ }^{2,3}$, Sural K. Ranamukhaarachchi' ${ }^{2}$,' Qi Yuan ${ }^{2,6 \bowtie}$, Mark Mayford ${ }^{2,3 凶}$

\author{
Affiliations: \\ ${ }^{1}$ Neurosciences Graduate Program, University of California San Diego, La Jolla, CA 92092, USA. \\ ${ }^{2}$ Department of Psychiatry, University of California San Diego, La Jolla, CA 92092, USA. \\ ${ }^{3}$ Department of Molecular and Cellular Neuroscience, Dorris Neuroscience Center, The Scripps \\ Research Institute, La Jolla, CA 92037, USA. \\ ${ }^{4}$ CAPES Foundation, Ministry of Education of Brazil, Brasília, DF 70040-020, Brazil. \\ ${ }^{5}$ Doctoral Program in Chemical and Biological Sciences, The Scripps Research Institute, La Jolla, \\ CA 92037, USA. \\ ${ }^{6}$ Division of Biomedical Sciences, Faculty of Medicine, Memorial University of Newfoundland, St. \\ John's, Newfoundland and Labrador A1B 3V6, Canada. \\ Corresponding authors: leominete@gmail.com; qi.yuan@med.mun.ca; mmayford@ucsd.edu
}

One Sentence Summary: Synaptic potentiation in the basolateral amygdala is necessary and sufficient for context fear learning.

\begin{abstract}
The nature and distribution of the synaptic changes that underlie memory are not well understood. We examined the synaptic plasticity behind context fear learning and found that conditioning produced potentiation of excitatory synapses specifically onto the basolateral amygdala neurons activated during learning. This synaptic potentiation lasted at least 7 days, and its disruption impaired memory recall. High frequency optogenetic stimulation of the CS and US-activated ensembles or biochemical induction of synaptic potentiation in US-responsive neurons alone was sufficient to produce a context fear association without prior associative training. These results suggest that plasticity of CS inputs onto US-responsive amygdala neurons is a necessary and sufficient step in forming context fear associations, and that context discrimination is determined by the CS-specific amygdala inputs activated during retrieval.
\end{abstract}




\section{Introduction}

Memories are thought to be mediated by synaptic changes induced by learning and distributed within the circuits controlling behavior. In contextual fear conditioning, lesion and pharmacological studies support a role for many brain areas, including the hippocampus, retrosplenial cortex, prefrontal cortex, and basolateral amygdala $(\mathrm{BLA})^{1-7}$; furthermore, studies using optogenetic activation of cfos labeled neuronal ensembles demonstrate that artificial reactivation of learning specific ensembles in each of these areas can induce an apparent recall event ${ }^{8-11}$. While these studies define the circuitry controlling the behavior and suggest a widely distributed network, they do not identify the sites of plasticity that are necessary for the memory. Previous studies of tone-cued fear conditioning have found that synapse-specific potentiation of auditory synaptic inputs to the lateral amygdala (LA) is necessary for auditory fear memory and auditory discrimination ${ }^{12,13}$, although not sufficient ${ }^{14}$. The role of amygdala plasticity in contextual learning is less well characterized. Interestingly, in many of the studies examining learning-induced plasticity in the LA/BLA, the behavioral training would be expected to produce a contextual fear memory in addition to tone conditioning, yet the synaptic changes observed were only dependent on tone-shock pairing contingencies $^{15-18}$. This could reflect either a lack of amygdala-based plasticity underlying contextfear associations or a sparse context representation that was not captured by recording from randomly targeted neurons.

\section{Results}

\section{Context conditioning induces circuit-specific synaptic potentiation in BLA}

To test whether context conditioning induces circuit-specific synaptic changes in the BLA, we used the c-fos-shEGFP transgenic mouse line (Fig. 1A), which expresses a short half-life EGFP under the control of the c-fos promoter ${ }^{19}$. Mice underwent associative fear conditioning and were either tested for memory 24 hours later or sacrificed after 90 minutes followed by whole-cell recordings in a BLA slice preparation (Fig. 1, B and C). As it is unknown how the contextual information reaches the amygdala, and given the likely distributive nature of such complex stimulus, synaptic responses of GFP+ (c-fos + ) and GFP- neurons were examined with targeted recordings in the BLA while stimulating internal capsule input fibers (Fig. 1D). As the role of the amygdala in tone fear conditioning has been extensively studied, we used tone presentations as a positive control in our experiments. We recorded $\alpha$-amino-3-hydroxy-5-methyl-4-isoxazolepropionic acid receptor (AMPAR) / N-methyl-D-aspartate receptor (NMDAR) ratios as a measure of excitatory synaptic 
strength $^{16,20}$, and found that fear conditioning produced an increase in AMPAR/NMDAR ratio that was limited to the GFP+ neurons (Fig. 1E), consistent with activity-dependent potentiation and as seen previously in the LA with tone conditioning ${ }^{12,13}$. However, unlike previous studies with blind recordings in respect to Fos expression ${ }^{15,16,18}$, we also observed an increase in AMPAR/NMDAR ratio during unpaired tone conditioning (i.e. conditioning to context only), suggesting synaptic potentiation occurs in BLA after context conditioning (Fig. 1E). The observed increase in AMPAR/NMDAR ratio is likely due to associative learning, and not simply derived from CS or US exposure, as a protocol that does not induce context conditioning, with overnight habituation to the context $^{21}$ (ON hab; Fig. 1C), fails to induce an AMPAR/NMDAR ratio increase (Fig. 1E), despite tagging a similar amount of cells with GFP (fig. S2, A and B). This suggests that the activation of immediate-early genes such as c-fos in a subset of BLA neurons does not correlate with learning. Although the AMPAR/NMDAR ratio is increased after context conditioning, GFP+ neurons do not differ in spike number, latency to spike, resting-membrane potential or paired pulse ratio (fig. S1).

To examine the persistence of plasticity in BLA neurons after context conditioning, we employed a double transgenic Arc-tTA/TetO-H2BGFP mouse line (Fig. 1F), in which a long-lasting histone-bound GFP is expressed in Arc+ cells. Active neurons were tagged with GFP at the time of learning and acute slices were prepared for whole-cell recordings 7 days later (Fig. 1G). The percentage of GFP+ cells were similar to c-fos-shEGFP transgenic mouse, used in previous experiments (fig. S2, A and B). We found that the AMPAR/NMDAR ratio increase in learningactivated neurons persists for at least 7 days (Fig. 1H), however potentiation was reversed in a group that received extinction training (fig. S2, C to F). Interestingly, re-conditioning animals that had undergone extinction reinstates the AMPAR/NMDAR ratio increase in the original GFP+ neuronal ensemble (fig. S2). Together, these results suggest that, following contextual fear conditioning, learning-induced synaptic potentiation in BLA neurons is circuit-specific, long-lasting, and is modulated by post-learning experience. 
bioRxiv preprint doi: https://doi.org/10.1101/2022.01.09.475530; this version posted January 11, 2022. The copyright holder for this preprint (which was not certified by peer review) is the author/funder, who has granted bioRxiv a license to display the preprint in perpetuity. It is made available under aCC-BY-NC 4.0 International license.
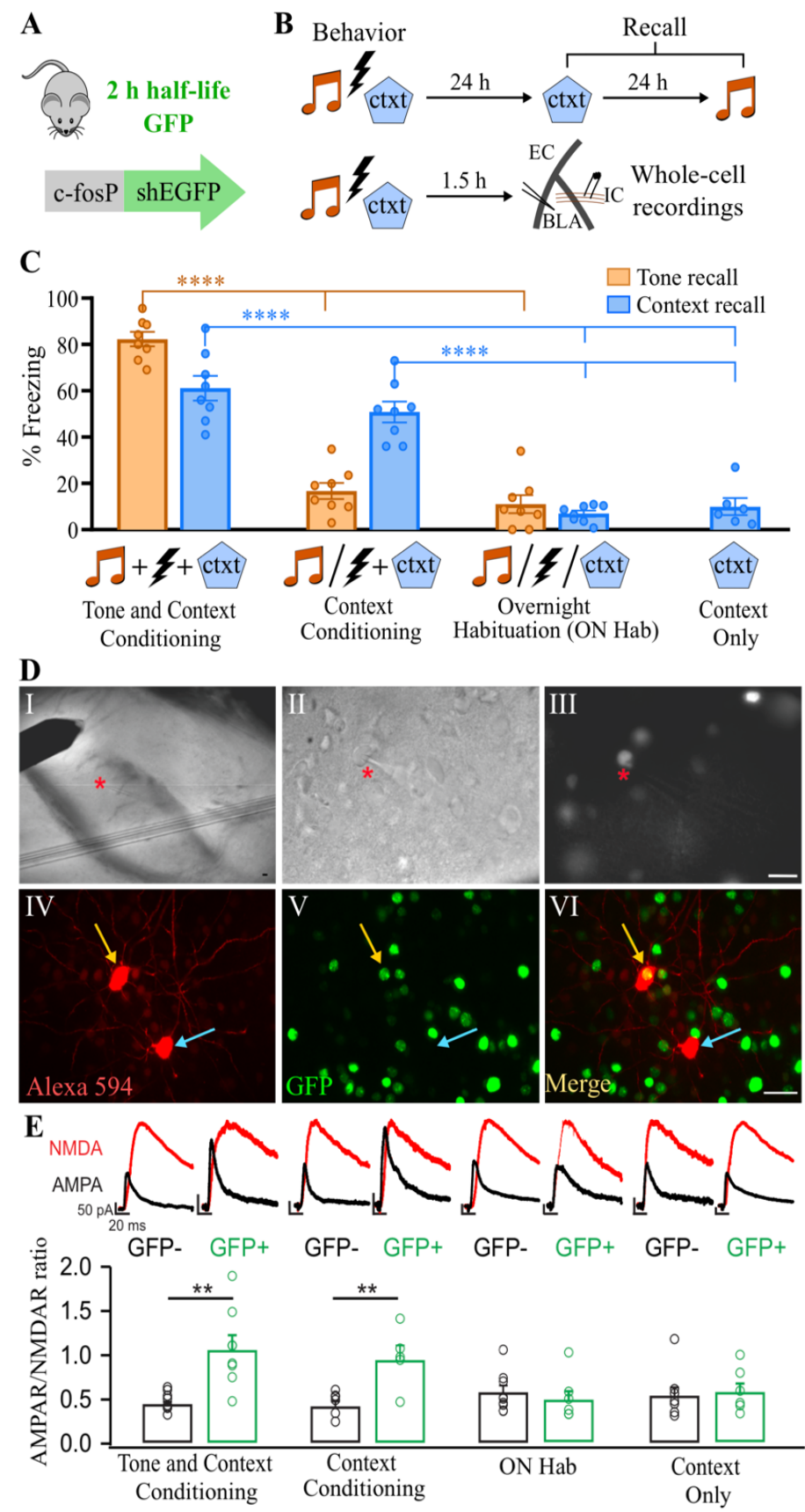

F

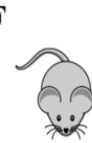

Long-lasting GFP

G

H
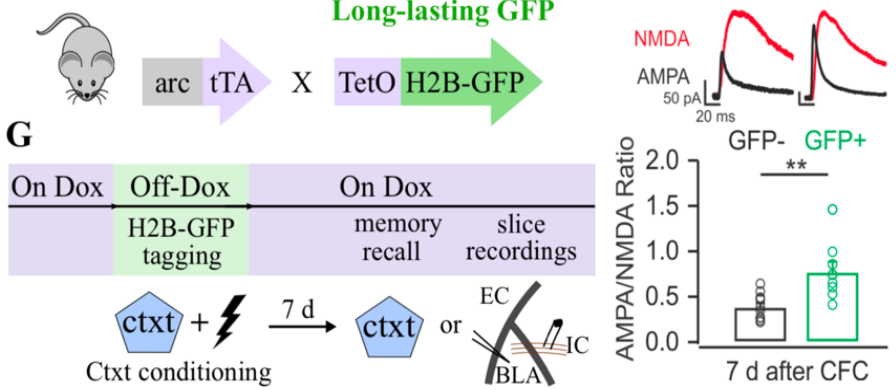

Fig. 1. Context learning induces long-lasting synaptic potentiation specifically in learningactivated BLA neurons. (A) The c-fos-shEGFP transgenic mouse, expressing a short half-life 
(sh)EGFP under the control of the c-fos promoter. (B) Experimental design in which the c-fosshEGFP mouse was either used for whole-cell recordings or memory recall tests after different behavior treatments. Ctxt: context; EC: external capsule; IC: internal capsule; BLA: basolateral amygdala. (C) Freezing response to the context (blue bars) and/or tone (orange bars) measured $24 \mathrm{~h}$ after correspondent behavior treatment. $\mathrm{N}=8$ per group, except in "Context only" group, in which $\mathrm{n}$ =6. (D) Whole-cell recording design. Panel I shows stimulating electrode over Internal Capsule fiber bundle. II and III illustrate ongoing whole-cell recording (red asterisk) of a GFP+ neuron. IVVI are confocal images of neighboring neurons filled with Alexa 594 during recordings: the GFP+ neuron is highlighted by a yellow arrow, while the GFP- is highlighted by a cyan arrow. Scale bars: $50 \mu \mathrm{m}$. These images were taken from a slice of an Arc-tTA/TetO-H2B-GFP mouse (F). (E) AMPAR/NMDAR ratio of GFP+ vs GFP- BLA neurons from acute brain slices of c-fos-shEGFP mice sac'ed $90 \mathrm{~min}$ after behavior treatment. An increase in AMPAR/NMDAR ratio was only observed after successful conditioning and was restricted to GFP+ neurons. $\mathrm{N}=6-9$ per group. (F) Arc-tTA x TetO-H2BGFP double transgenic mouse, in which the tetracycline transactivator (tTA) was knocked in the Arc gene and controls the expression of the long-lasting histone-bound GFP. (G) Experimental design illustrating the tagging window of the Arc-tTA/TetO-H2B-GFP mouse line, controlled by the presence of Doxycycline (Dox) in the food. Tagged neurons were recorded 7 days after. Correspondent behavior result can be found on fig. S2C. (H) Specific AMPAR/NMDAR ratio increase in GFP+ neurons following conditioning is maintained for at least 7 days. CFC: Contextual fear conditioning. $\mathrm{N}=8-9$ per group. ${ }^{* *} \mathrm{P}<0.01$, $* * * * \mathrm{P}<0.0001$, one-way ANOVA with Tukey test (C), unpaired t test $[(\mathrm{E})$ and $(\mathrm{H})]$. Graph bars show mean $+/-$ SEM.

\section{Learning-induced synaptic potentiation in BLA is necessary for memory recall.}

To test whether this synaptic potentiation represents a necessary component of the context fear memory trace, we used a biochemical approach to reverse the plasticity. CaMKII $\alpha$ is a critical component in the induction of synaptic plasticity, with mutant forms of the kinase known to either induce long term-potentiation (LTP) or long-term depression (LTD) ${ }^{22}$. We used a c-fos driveninducible Cre recombinase mouse line (FDC mouse) ${ }^{23}$ combined with AAV-based gene delivery to introduce the LTD-inducing CaMKII $\alpha$-T286D (CK2-D) mutant ${ }^{22}$ into BLA neurons activated with learning (Fig. 2A). In the FDC mouse, Cre-recombinase is regulated by trimethoprim(TMP)induced protein stabilization, providing a ligand-gated time window for Cre activity of approximately 4 hours ${ }^{23,24}$. Using this system, we induced expression of CK2-D and a GFP marker in BLA neurons activated during fear conditioning (Fig. 2, B and C) and tested the memory on the same day, before significant CK2-D expression, and 7 days after CK2-D induction (Fig. 2D), when it has reached peak expression (fig. S3, A to E).

CK2-D expression in c-fos+ BLA neurons tagged during training reverses the long-lasting increase in AMPAR/NMDAR ratio [(Fig. 2E), compare with Figure 1H], and reduces the amplitude 
of mini-EPSCs without affecting their frequency, paired-pulse ratio or intrinsic excitability (fig. S4 and table 1), suggesting specific reversal of learning-induced synaptic potentiation. Behaviorally, CK2-D expression in c-fos + BLA neurons tagged during training impairs both tone and context memories, while the control group, injected with wild-type CaMKII $\alpha$ (WT-CK2) AAV had intact memories, suggesting the effect is not due to overexpression of CaMKII $\alpha$ (Fig. 2, F and G). Memory recall tests four weeks after training indicate that the CK2-D induced memory impairment is longlasting (fig. S5). Staining for endogenous c-fos during memory retrieval showed that the reactivation of neurons tagged during training (GFP+), which has been shown to correlate with memory retrieval 19,25, was impaired by CK2-D expression (Fig. 2, H and I), suggesting an inability to access these ensembles during recall.

A

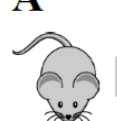
c-fos DD-Cre

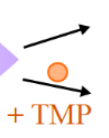

C $\mathrm{r} / \mathrm{D}$ 'J Degradation

Cre DD $\bigcirc$ Expression

\section{CaMKIIaP} rAAV-DJ

\section{H2B-GFP 2A Wt or mut. CaMKII $\alpha$}

D

WT-CK2 or CK2-D AAV inj Tone and Context

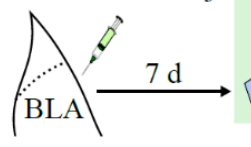
ctxt

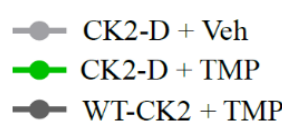

F Tone Recall

$\mathbf{J}$

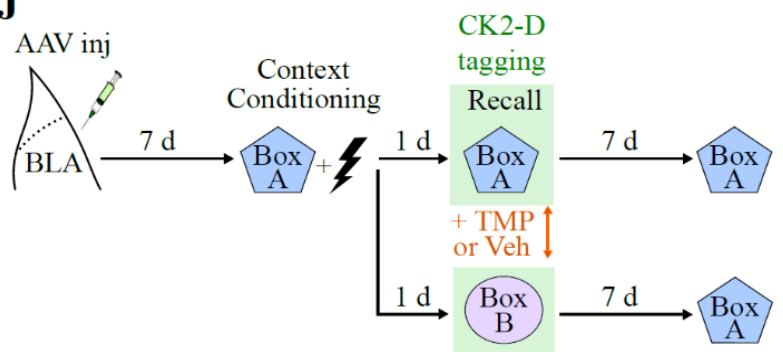

G
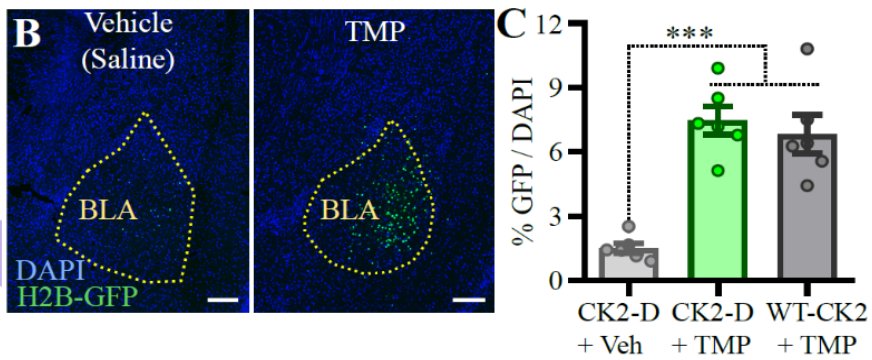

$\mathbf{E}$

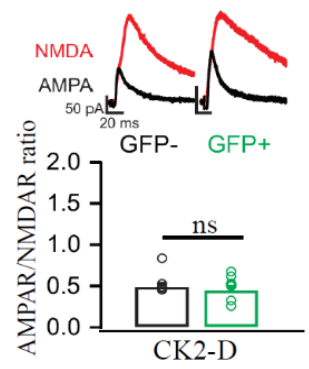

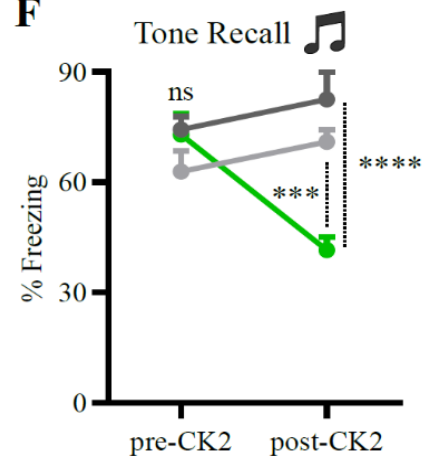
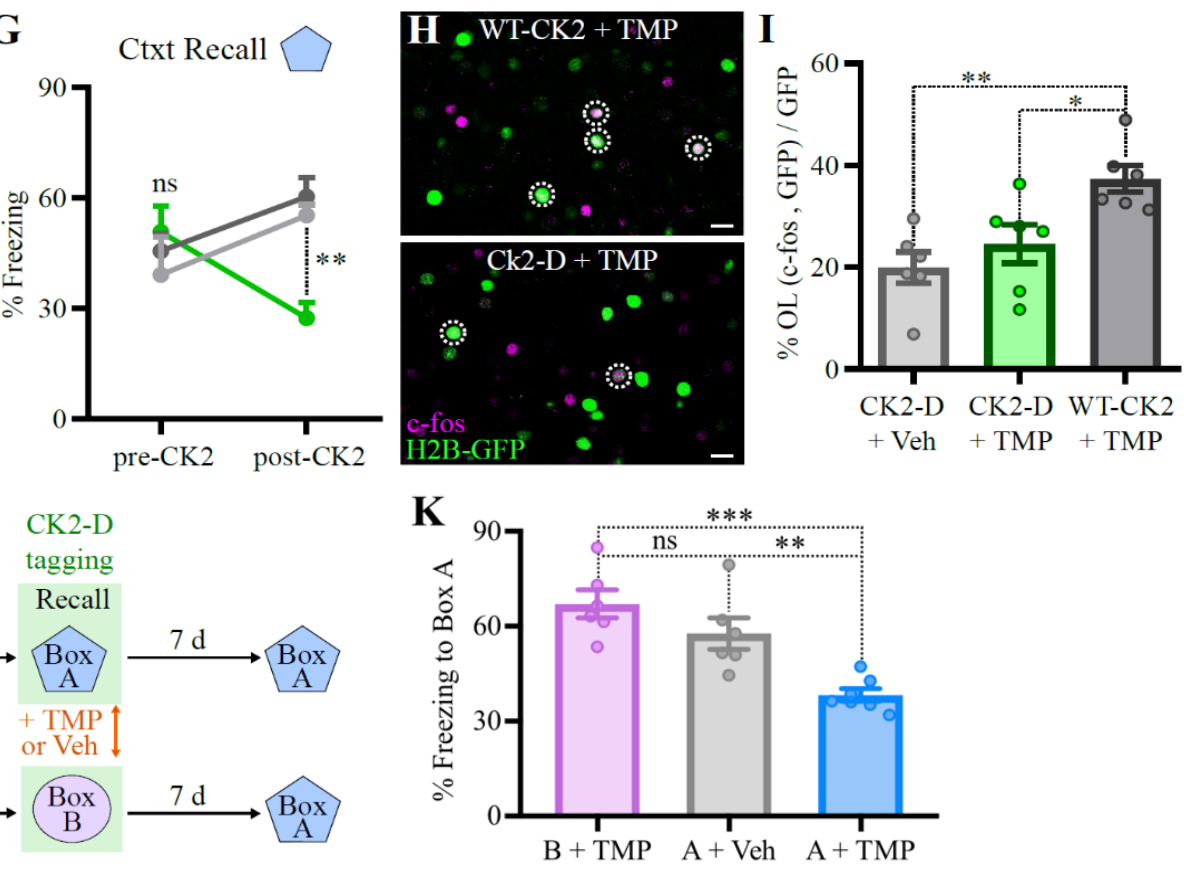
Fig. 2. Disruption of learning-induced synaptic potentiation in BLA impairs long-term memory. (A) rAAV-DJ carrying a DIO-(wild-type or mutant) CaMKII $\alpha-2 \mathrm{~A}-\mathrm{H} 2 \mathrm{BGFP}$ construct was injected bilaterally in the BLA of c-fos-DD-Cre (FDC) mice, in which Cre-recombinase is fused to a destabilizing domain (DD), which leads to protein degradation. The drug trimethoprim lactate (TMP) stabilizes the complex, allowing Cre to activate the CaMKII $\alpha$ construct along with the H2BGFP marker in c-fos + BLA neurons. (B) and (C) Representative coronal BLA sections showing H2B-GFP induction 14 days after TMP or vehicle (saline) IP injection, quantified in (C). $\mathrm{N}=6$ mice per group. Scale bar: $200 \mu \mathrm{m}$. (D) Experimental design to test whether CK2-D impairs memory recall. (E) CK2-D expression in c-fos + BLA neurons tagged during context conditioning reverses learning-induced AMPAR/NMDAR ratio increase. No tone conditioning/exposure was performed in order to allow for clearer interpretation of the effect of CK2-D expression on context conditioninginduced plasticity. $\mathrm{N}=7-8$ neurons per group. (F) and (G) CK2-D expression in c-fos + BLA neurons tagged during training impairs long-term memory, as opposed to WT-CK2. N=6 mice per group. (H) and (I) Representative coronal BLA sections showing overlap between activity markers during training (H2B-GFP) and context recall [(c-fos IHC), (H)], quantified in (I). CK2-D reversal of synaptic potentiation impaired reactivation of learning-induced neurons. OL: overlap. $\mathrm{N}=6$ mice per group. Scale bar: $20 \mu \mathrm{m}$. (J) Experimental design to test context specificity. (K) CK2-Dmediated memory impairment is specific to tagged context. $\mathrm{N}=6-7$ per group. $* \mathrm{P}<0.05, * * \mathrm{P}<0.01$, $* * * \mathrm{P}<0.001, * * * * \mathrm{P}<0.0001$, ns, not significant; one-way ANOVA with Tukey test [(C), (I) and $(\mathrm{K})$ ], unpaired t-test (E), or two-way RM ANOVA with Tukey test [(F) and (G)]. Graphs show mean $+/-$ SEM.

To test whether CK2-D-mediated memory impairment was context-specific, we genetically tagged c-fos neuronal ensembles activated in either the conditioning box (box A) or a novel box (box B) one day after training (Fig. 2J). Recall in box A 7 days later showed that the memory was impaired only when CK2-D was expressed in box A activated ensembles (Fig. 2K), despite a similar proportion of labeled neurons in both groups (fig. S6). In summary, these results suggest that synaptic potentiation in learning-activated BLA neurons is a necessary component of the contextual fear engram, and that this plasticity is context specific.

\section{Learning-induced synaptic potentiation in BLA is sufficient for context-fear association.}

Next, we sought to test whether synaptic potentiation alone in learning-activated BLA neurons is sufficient to produce a context fear association. We attempted to produce a de novo associative fear memory by inducing plasticity in neurons activated independently by the conditioned-stimulus (CS) and the unconditioned-stimulus (US), as shown in Figure 3. We used the rAAV2-retro serotype ${ }^{26}$ to introduce a Cre-dependent ChR2(H134R)-EYFP (ChR-retro) into the BLA of FDC mice to allow genetic tagging of CS and US-activated ensembles in both local and 
input neurons (Fig. 3A). The co-injection of an rAAV-DJ containing a Cre-dependent nuclear marker confirmed that the injection was restricted to the BLA (fig. S7, A to C). In addition to the BLA, we found significant ChR-retro expression in (from the most intense to the least) the prefrontal cortex (PFC, including prelimbic, infralimbic and mediorbital cortices), the entorhinal cortex (EC, both lateral and medial portions), the paraventricular nucleus of the thalamus (PVT), the ectorhinal and perirhinal cortices (Ect-PRh), and the insular cortex (InC) (Fig. 3B and fig. S7). There was inconsistent labeling of the auditory cortex $(\mathrm{AuC})$ when the injection hit more dorsal parts of BLA (Fig. 3B). Very little to no labeling was found at some known BLA input regions such as the ventral hippocampus (vHPC) ${ }^{27}$, the medial geniculate nucleus (MGN) and the periaqueductal gray (PAG), suggesting the rAAV2-retro serotype infects cortico-amygdalar inputs more effectively (fig. S7E). This specificity may have derived from the directed evolution and selection process used in its development, favoring the labeling of cortical projections ${ }^{26}$.

In order to test if the potentiation of either CS or US inputs, or both, is sufficient to produce a de novo associative fear memory, we used an unpaired training protocol in which the CS (box $\mathrm{B}+$ tone) and US (shock) were presented separately on different days to avoid any natural learned association (Fig. 3, C and D). To induce synaptic potentiation in BLA inputs, we delivered $100 \mathrm{~Hz}$ light pulses through optic fibers implanted bilaterally over the BLA. This stimulation protocol has been shown to induce LTP in several brain regions ${ }^{12,14,28,29}$. The optogenetic stimulation at $100 \mathrm{~Hz}$ in the BLA produced a de novo associative fear memory to box B only when both CS and US inputs were tagged, while no memory was formed when either one of them was tagged alone (Fig. 3E). This artificial memory association was context-specific, as no memory to an untagged box (box C) was formed. No auditory fear association was formed which may be due to insufficient ChR-retro expression in auditory regions (Fig. S8D). Using a double transgenic line that expresses the ChRvariant $\mathrm{ChEF}$ in c-fos + neurons broadly throughout the brain ${ }^{8}$, we found that the same optogenetic stimulation protocol at $100 \mathrm{~Hz}$ produces a de novo fear association to both the tone and context (fig. S9). The optogenetic stimulation did not cause any noticeable performance alterations in anxiety tests, including the elevated plus-maze, the open field, and marble burying tests (fig. S8, A to C).

One model of fear learning posits that it occurs through the potentiation of CS inputs onto amygdala neurons ${ }^{30}$, suggesting that the potentiation of CS inputs alone would be sufficient to generate a de novo contextual fear memory association, which did not occur in our hands (Fig. 3, D and E, "CS" group, in blue). One possible explanation is that the indiscriminate potentiation of CS inputs might result in no clear behavioral outcome ${ }^{14}$, as BLA is a critical component of behaviors of 
distinct emotional valence ${ }^{31}$. Indeed, BLA neurons tagged during box exposure (CS) project less heavily to the central amygdala $(\mathrm{CeA})$, a known fear center, and more heavily to the nucleus accumbens (NAc) core than BLA neurons tagged during context conditioning (CS + US) (fig. S10), which suggests that different neuronal populations are activated by the CS alone compared to CS + US ${ }^{32}$. In our setup, the tagging of US neurons might be crucial to direct the optogenetic manipulation towards aversive behaviors.

To test whether we could reverse the artificial memory association to box B, we used $1 \mathrm{~Hz}$ optogenetic stimulation (Fig. 3C), as it has been shown to induce LTD in auditory inputs to LA ${ }^{13,14}$. We found that, after $1 \mathrm{~Hz}$ light pulses, the freezing levels to box B returned to baseline, suggesting reversal of the artificial context memory (Fig. 3F). Interestingly, the memory to box A (tagged with the US, Fig. 3C) was not significantly affected (Fig S8E), which suggests that the natural recall may also occur through routes that were insufficiently tagged by ChR-retro, including vHPC ${ }^{27}$ and subcortical regions (fig. S7C). Despite the need for tagging both CS and US inputs for de novo memory generation, we find that the tagging of the US neurons alone is sufficient to lead to lightinduced freezing during prolonged optogenetic stimulation at $20 \mathrm{~Hz}$ in a novel box (Fig. 3G). This stimulation protocol induces enhanced c-fos expression in BLA, which overlaps with the US-tagged cells (fig. S11).

A

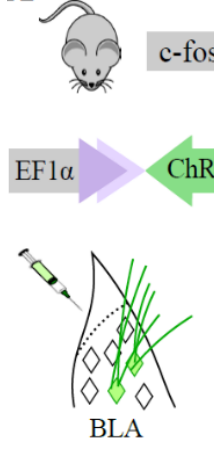

B

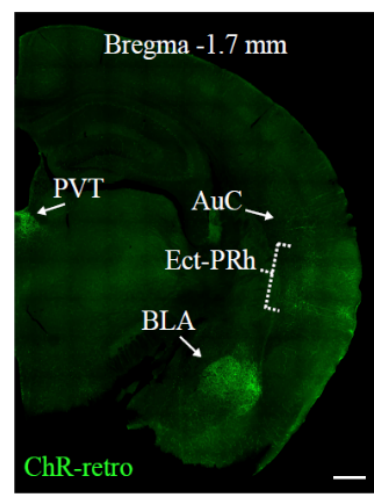

Labeling of BLA neurons and their presynaptic partners

C

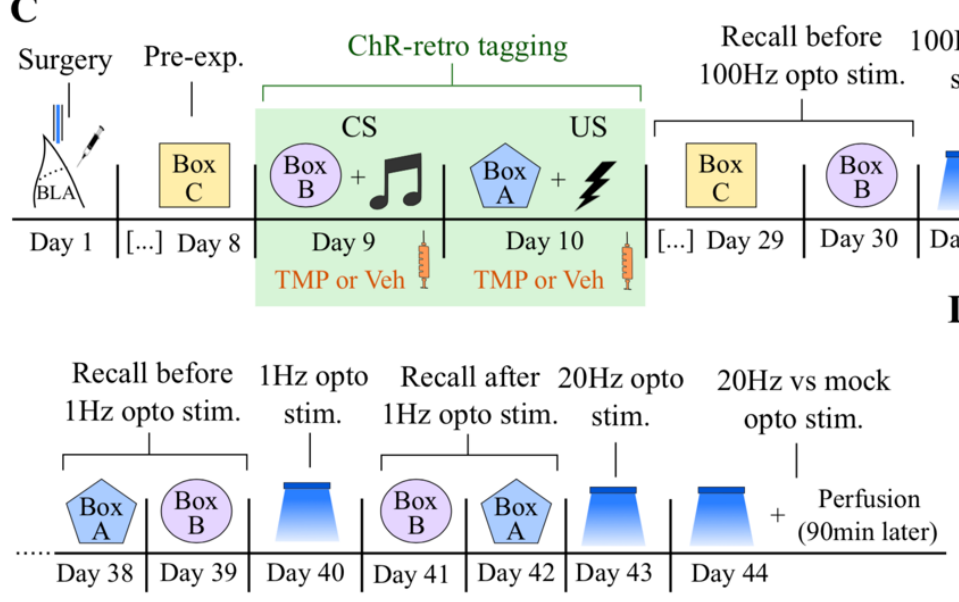

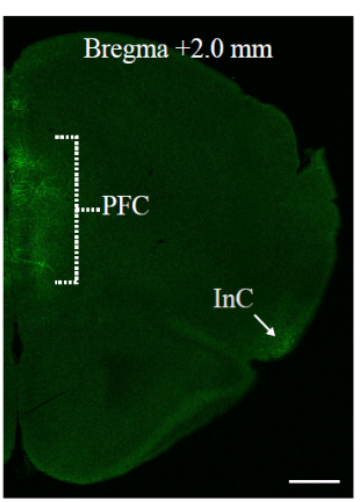

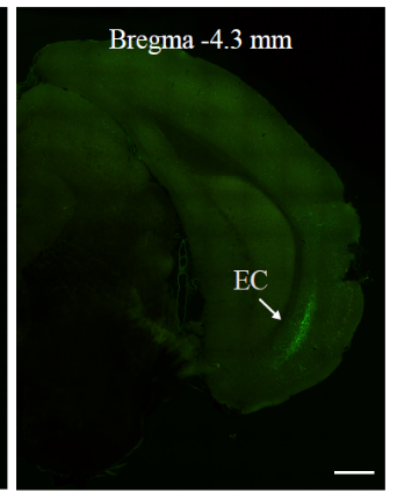

Recall after $100 \mathrm{~Hz}$ opto stim.
Anxiety

tests
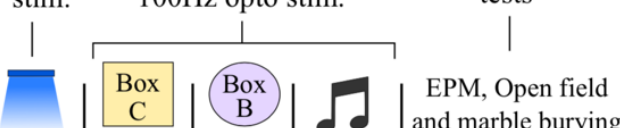
and marble burying

\begin{tabular}{|c|c|c|}
\hline $\begin{array}{c}\text { CS } \\
\text { Box } \\
B\end{array}$ & $\begin{array}{c}\text { US } \\
\widehat{B \text { Box }}+\boldsymbol{Y}\end{array}$ & $\begin{array}{l}\text { Experimental } \\
\text { groups }\end{array}$ \\
\hline Veh & Veh & $\multimap \quad$ Veh \\
\hline TMP & Veh & $-\infty$ \\
\hline Veh & TMP & $\rightarrow \quad$ US \\
\hline TMP & TMP & $\rightarrow \mathrm{CS}+\mathrm{US}$ \\
\hline
\end{tabular}


$\mathbf{E}$

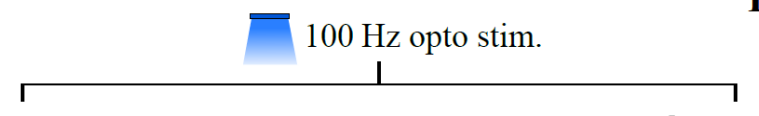

Box C recall
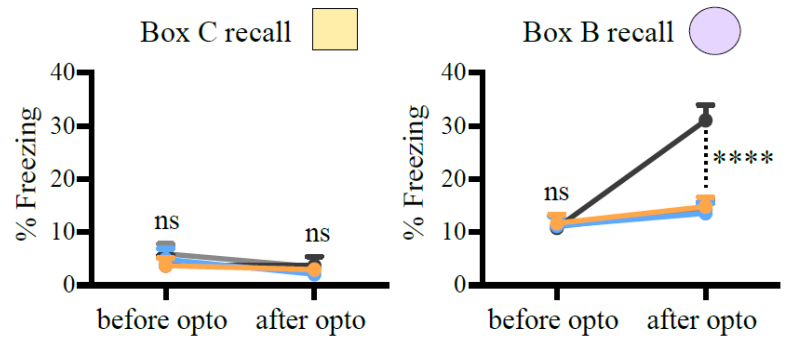

$\mathbf{F}$
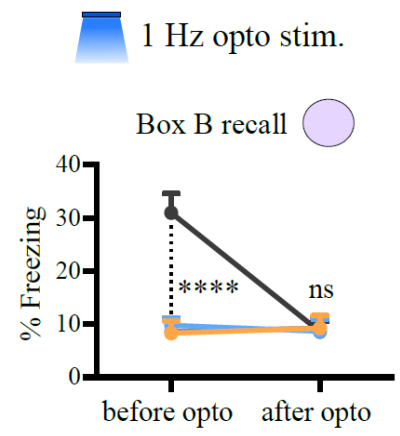

G
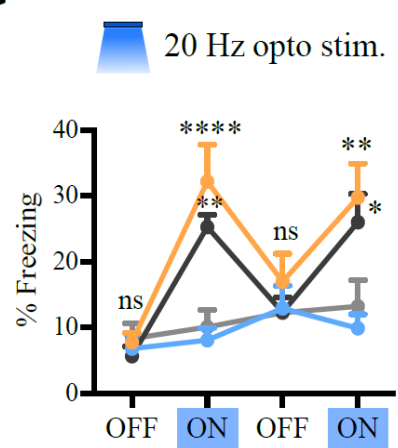

Fig. 3. $100 \mathrm{~Hz}$ optogenetic stimulation of both CS+ and US+ tagged neurons in BLA and their inputs is sufficient to create a de novo contextual aversive memory. (A) AAV2-retro carrying a DIO-ChR2(H134R)-eYFP (ChR-retro) construct was bilaterally injected in the BLA of FDC mice, leading to expression of ChR-retro in tagged BLA local and input neurons. (B) Coronal sections showing the expression pattern of ChR-retro after injection in BLA and tagging during exposure to both CS and US (see C and D). The brain regions with the most significant expression are labeled. AuC: Auditory Cortex (low expression); BLA: Basolateral amygdala; EC: Entorhinal cortex, including lateral and medial regions; Ect-PRh: Ectorhinal and Perirhinal cortices; InC: Insular cortex; PFC: Prefrontal cortex, including prelimbic, infralimbic and mediorbital regions; PVT: Paraventricular nucleus of thalamus. Scale bar: $500 \mu \mathrm{m}$. (C) Experimental design for optogeneticmediated de novo memory generation and reversal. (D) Experimental groups and their respective tagging regimes, with either TMP or Veh IP injected 20 min after each behavior exposure. $\mathrm{N}=8$ mice per group. (E) $100 \mathrm{~Hz}$ optogenetic stimulation of BLA local and input neurons generates an artificial memory that is specific to the tagged context (Box B) only when both CS and US are tagged. (F) $1 \mathrm{~Hz}$ optogenetic stimulation reverses the artificially created memory to box B. (G) Freezing behavior during $20 \mathrm{~Hz}$ light stimulation occurs only when the US is tagged. Opto stim.: Optogenetic stimulation. $* * * * \mathrm{P}<0.0001, * * \mathrm{P}<0.01 ;{ }^{*} \mathrm{P}<0.05$, ns: not significant. Two-way RM ANOVA with Tukey test. Graph bars show mean +/- SEM.

One caveat of optogenetic stimulation is the possible off-target effects both upstream and downstream of the target area due to the stimulation-driven circuit response ${ }^{33}$. Therefore, just based on the optogenetic experiments shown in Fig. 3, we cannot conclude that the potentiation of BLA synapses is sufficient to form the context-fear association. To circumvent this limitation, we developed a strategy to biochemically induce LTP only in the US neurons of the BLA with the expression of the triple mutant CamKII $\alpha$ T286D-T305A-T306A (CK2-DAA), which has been shown to induce LTP in hippocampal organotypic slices ${ }^{22}$. Using a similar approach to that described in Figure 2, we bilaterally injected an AAV containing a CRE-dependent construct expressing CK2-DAA and a nuclear GFP marker into the BLA of FDC mice (Fig. 4A). To test whether CK2-DAA could induce LTP in BLA neurons in the absence of shock, we tagged neurons 
during a novel box exposure, and 7 days later, prepared slices for whole-cell recordings (Fig. 4B). Indeed, the CK2-DAA+ (GFP+) neurons exhibited an increase in AMPAR/NMDAR ratio when compared to the neighboring GFP- neurons (Fig. 4C), without affecting their intrinsic excitability (Table S2). This increase in AMPAR/NMDAR was not seen with overexpression of wild-type CaMKII $\alpha$ (WT-CK2) (Fig. 4C).

If plasticity in BLA alone is sufficient to create context fear associations, which synapses need to be potentiated? To address that question, we used a similar unpaired training protocol to the one described in Figure 3, in which CS and US were tagged independently on different days (Fig. 4, $\mathrm{D}$ and E). CK2-DAA expression is insignificant one day after TMP induction, but substantial at 7 days (fig. S3, F to $\mathrm{H}$ ). We found that the potentiation of BLA neurons tagged during US exposure alone is sufficient to drive both tone and context-mediated aversive associations, and that this is generalized to multiple contexts. (Fig. 4, F to H). Despite enhanced cue-induced freezing levels, no significant changes in locomotion or common anxiety behaviors were observed during elevated plusmaze, open field or marble burying tests (fig. S12, A to C). To rule out the possibility that any new fear memories were due to the formation of hybrid associations with the natural memory to box $\mathrm{A}^{34}$, we included a control in which the animals were habituated overnight to box A (ON Hab) before the exposure to the shock to prevent any conditioning ${ }^{21}$ (Fig. 1, C and E). Still, we observed generalized context fear associations (Fig. 4, F to H, purple line), even though the freezing to box A was reduced to a level similar to untrained boxes B and C (fig. S12D). Staining for c-fos induction during exposure to box $\mathrm{C}$ showed that CK2-DAA expression promotes the reactivation of neurons that were tagged during US exposure (GFP+) relative to neurons tagged with a WT-CK2 control (Fig. 4, I to J). This result suggests that the activation of US neurons facilitated by CK2-DAA induced-synaptic potentiation drives generalized context induced fear. 


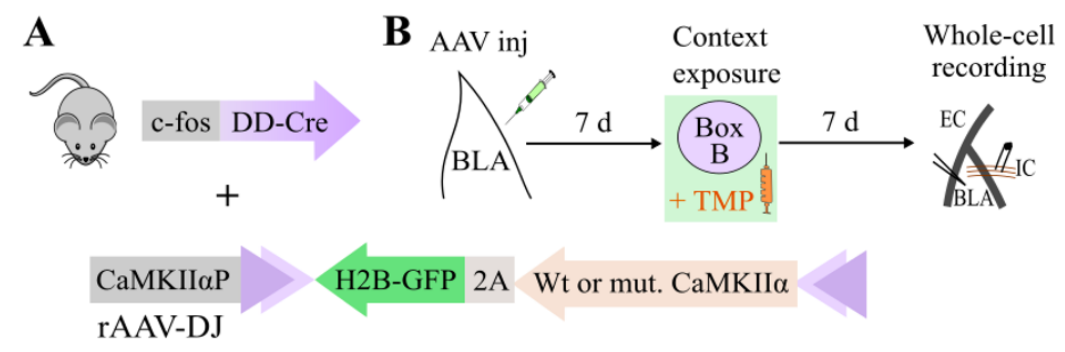

D
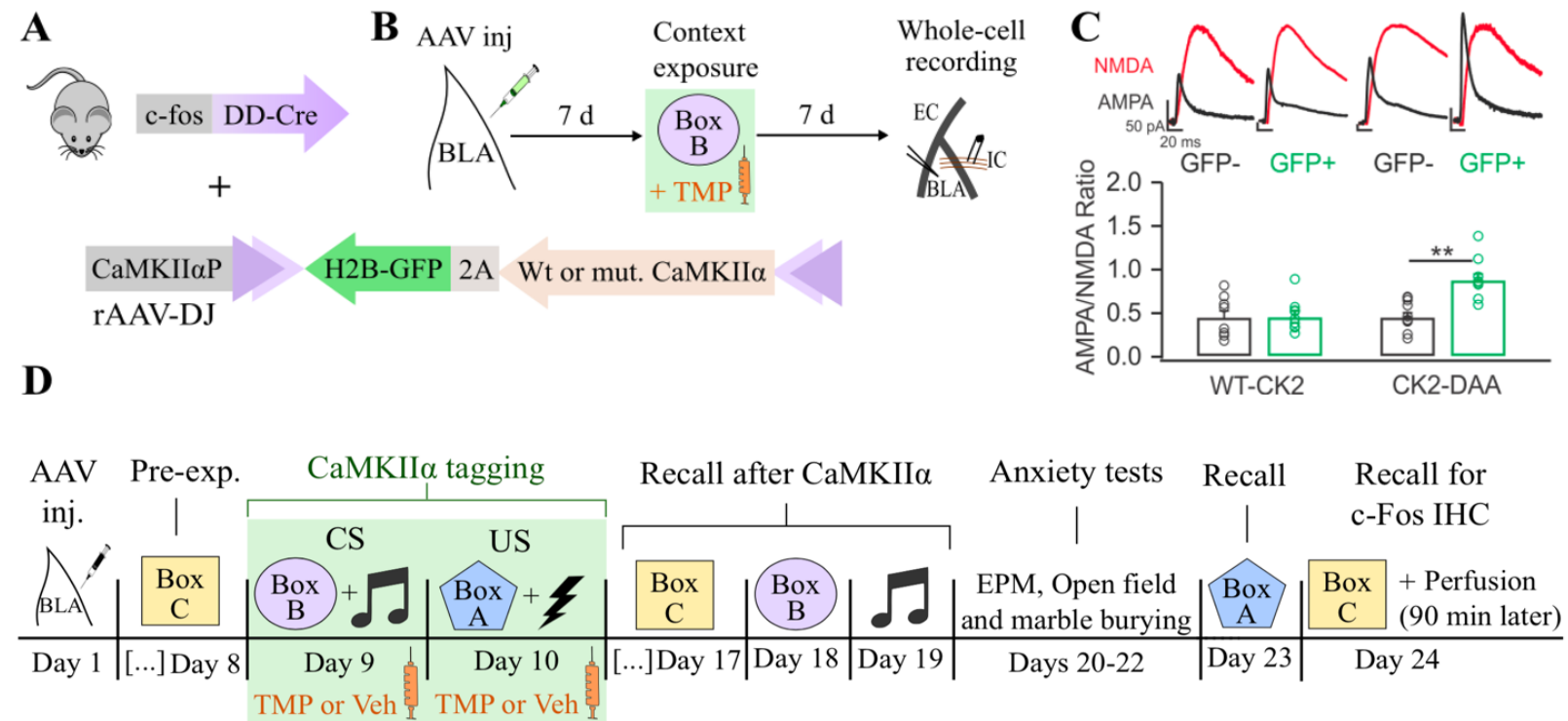

$\mathbf{E}$

\begin{tabular}{|c|c|cc|}
\hline $\begin{array}{c}\text { CS } \\
\text { Box }\end{array}$ & \multicolumn{2}{|c|}{$\begin{array}{c}\text { US } \\
\text { Box } \\
\text { A }\end{array}$} & \multicolumn{2}{|c|}{$\begin{array}{c}\text { Experimental } \\
\text { groups }\end{array}$} \\
\hline Veh & Veh & $-\bullet$ & Veh \\
\hline TMP & Veh & $-\bullet$ & CS \\
\hline Veh & TMP & $-\bullet$ & WT-CK2 \\
\hline Veh & TMP & - & ON Hab \\
\hline Veh & TMP & - & US \\
\hline TMP & TMP & - & CS + US \\
\hline
\end{tabular}

$\mathbf{F}$

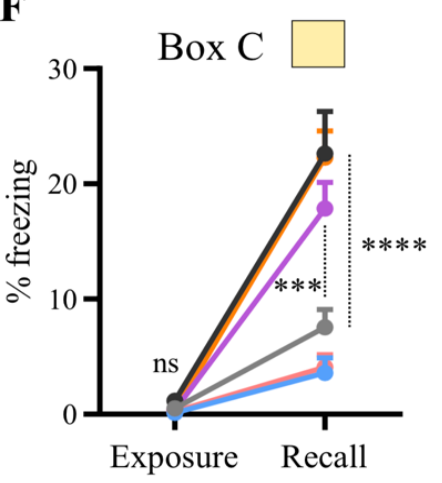

G

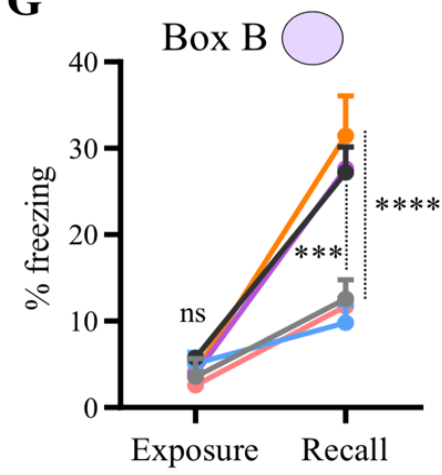

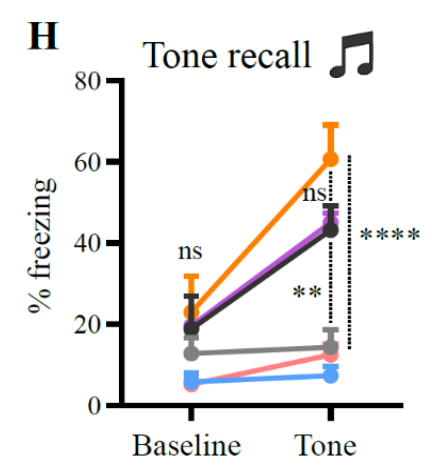
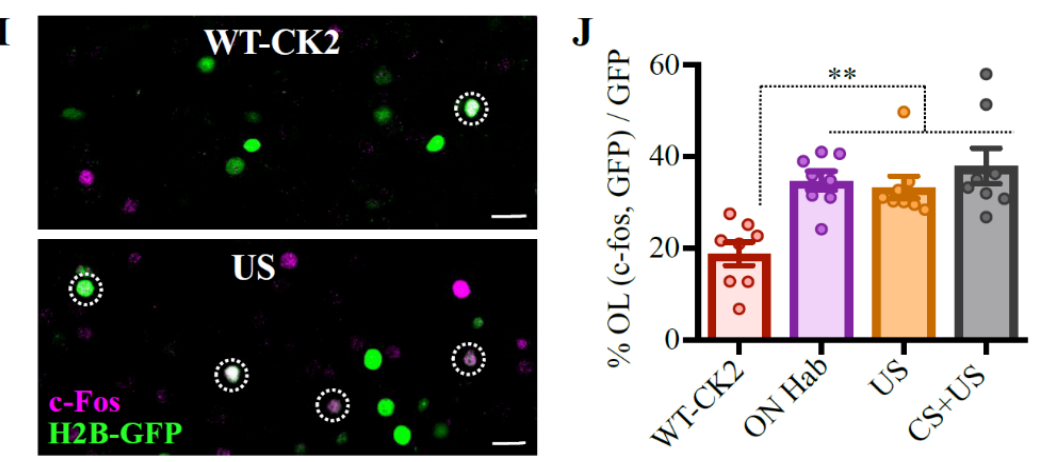

Fig. 4. Biochemical induction of synaptic potentiation in US+ BLA neurons is sufficient to generate long-term aversive memories. (A) rAAV-DJ carrying a DIO-CK2-2A-H2BGFP construct was injected bilaterally in the BLA of FDC mice. (B) Experimental design to test whether CK2-DAA induces synaptic potentiation in the BLA in the absence of US exposure. (C) CK2-DAA expression induces increase in AMPAR/NMDAR ratio, whereas WT-CK2 overexpression does not affect it. (D) Experimental design to test whether CK2-DAA mediated-potentiation creates a de novo memory association. (E) Experimental groups and their respective tagging regimes, with either TMP or Veh IP injected 20 min after each behavior exposure. Mice in the ON hab group were kept overnight in box A before shock exposure to prevent any conditioning (Fig. 1C). $\mathrm{N}=8$ mice per group, except in 
the Veh group, where $\mathrm{N}=7$. $(\mathbf{F})$ to $(\mathbf{H}) \mathrm{CK} 2-\mathrm{DAA}$ potentiation of US neurons led to indiscriminate fear associations, including the untagged box C (F). (I) and (J) Representative coronal BLA sections showing overlap between activity markers during exposure (H2B-GFP) and recall to the untagged box C [(c-fos IHC), (I)], quantified in (J). OL: overlap. Scale bar: $20 \mu \mathrm{m}$. ${ }^{*} * \mathrm{P}<0.01, * * * \mathrm{P}<0.001$, $* * * * \mathrm{P}<0.0001$, ns, not significant; unpaired t-test $(\mathrm{C})$, two-way RM ANOVA with Tukey test $[(\mathrm{F})$ to $(\mathrm{H})$ ] or one-way ANOVA with Tukey test $(\mathrm{J})$. Graphs show mean +/- SEM.

\section{Discussion}

Together, our results show that the essential underlying cellular plasticity for forming context fear associations is the potentiation of synaptic inputs onto US responsive neurons in the BLA that in turn project selectively to the central amygdala and the fear output circuit ${ }^{35}$. This suggests that even for hippocampal dependent tasks such as context recognition, which involve the integration of multimodal sensory cues, the fear association takes place in the amygdala and the specificity of the response is likely determined by the nature of the inputs activated during learning and retrieval. In fear conditioning, it is the aversive shock that is actually "recalled", as the animals are present in the identical context box during both learning and retrieval. According to this view, the role of hippocampal and cortical structures is to respond to the context cues by recruiting the same neuronal ensembles from the original context exposure during learning, with fear being expressed by the increased synaptic plasticity of BLA inputs and subsequent activation of the US-linked fear circuit.

Using a cellular tagging approach to study learning-induced plasticity, we were able to show that context extinction reverses the synaptic potentiation established during the original learning (fig. S2). Nevertheless, our findings do not preclude the occurrence of extinction-specific synaptic plasticity elsewhere in the circuit, as other studies have demonstrated ${ }^{36,37}$. Instead, our work suggests that extinction might involve not only new learning but also unlearning of the original memory. Understanding how these mechanisms cooperate during extinction, and which parameters promote unlearning, such as the optimal time window for this process ${ }^{38}$, might provide important lessons for the reduction of relapse after exposure therapy in a clinical setting ${ }^{39}$.

Previous lesion and pharmacological manipulations of various hippocampal and cortical structures have been shown to reduce context fear conditioning. This may reflect altered processing of contextual information preventing reactivation of the appropriate BLA inputs. Since our approach involved delivery of both the CS and US in an unpaired manner to tag appropriate ensembles, it is possible that these stimuli individually produce plasticity at other sites within the circuit that contribute to the learning. Nevertheless, the plasticity critical for forming the Pavlovian association 
seems to be localized in the BLA. Further studies could take advantage of the tagging strategy described in this work to dissect the precise synaptic mechanisms behind the CS-US association, such as the role of homosynaptic and heterosynaptic plasticity ${ }^{40-42}$, the subtypes of AMPARs involved $^{20,38}$, among others.

Synaptic plasticity found elsewhere in the hippocampus and other cortical regions during fear conditioning might be more involved in context encoding e.g. creating more reliable ensemble reactivation upon context reexposure. This hippocampal/cortical plasticity is likely to be particularly important in tasks that involve subtle context discrimination or complex spatial navigation, rather than simple place recognition.

\section{References}

1. Corcoran, K. A. et al. NMDA receptors in retrosplenial cortex are necessary for retrieval of recent and remote context fear memory. J Neurosci 31, 11655-11659 (2011).

2. Frankland, P. W., Bontempi, B., Talton, L. E., Kaczmarek, L. \& Silva, A. J. The Involvement of the Anterior Cingulate Cortex in Remote Contextual Fear Memory. Science 304, 881-883 (2004).

3. Gale, G. D. et al. Role of the Basolateral Amygdala in the Storage of Fear Memories across the Adult Lifetime of Rats. J. Neurosci. 24, 3810-3815 (2004).

4. Kim, J. J. \& Fanselow, M. S. Modality-specific retrograde amnesia of fear. Science 256, 675677 (1992).

5. Lesburguères, E. et al. Early Tagging of Cortical Networks Is Required for the Formation of Enduring Associative Memory. Science 331, 924-928 (2011).

6. Phillips, R. G. \& LeDoux, J. E. Differential contribution of amygdala and hippocampus to cued and contextual fear conditioning. Behav. Neurosci. 106, 274-285 (1992).

7. Quinn, J. J., Loya, F., Ma, Q. D. \& Fanselow, M. S. Dorsal hippocampus NMDA receptors differentially mediate trace and contextual fear conditioning. Hippocampus 15, 665-674 (2005).

8. Cowansage, K. K. et al. Direct Reactivation of a Coherent Neocortical Memory of Context. Neuron 84, 432-441 (2014).

9. Gore, F. et al. Neural Representations of Unconditioned Stimuli in Basolateral Amygdala Mediate Innate and Learned Responses. Cell 162, 134-145 (2015).

10. Kitamura, T. et al. Engrams and circuits crucial for systems consolidation of a memory. Science 356, 73-78 (2017).

11. Liu, X. et al. Optogenetic stimulation of a hippocampal engram activates fear memory recall. Nature 484, 381-385 (2012).

12. Abdou, K. et al. Synapse-specific representation of the identity of overlapping memory engrams. Science 360, 1227-1231 (2018). 
13. Kim, W. B. \& Cho, J.-H. Encoding of Discriminative Fear Memory by Input-Specific LTP in the Amygdala. Neuron 95, 1129-1146.e5 (2017).

14. Nabavi, S. et al. Engineering a memory with LTD and LTP. Nature 511, 348-352 (2014).

15. McKernan, M. G. \& Shinnick-Gallagher, P. Fear conditioning induces a lasting potentiation of synaptic currents in vitro. Nature 390, 607-611 (1997).

16. Namburi, P. et al. A circuit mechanism for differentiating positive and negative associations. Nature 520, 675-678 (2015).

17. Rogan, M. T., Stäubli, U. V. \& LeDoux, J. E. Fear conditioning induces associative long-term potentiation in the amygdala. Nature 390, 604-607 (1997).

18. Tsvetkov, E., Carlezon, W. A., Benes, F. M., Kandel, E. R. \& Bolshakov, V. Y. Fear conditioning occludes LTP-induced presynaptic enhancement of synaptic transmission in the cortical pathway to the lateral amygdala. Neuron 34, 289-300 (2002).

19. Reijmers, L. G., Perkins, B. L., Matsuo, N. \& Mayford, M. Localization of a Stable Neural Correlate of Associative Memory. Science 317, 1230-1233 (2007).

20. Rumpel, S., LeDoux, J., Zador, A. \& Malinow, R. Postsynaptic Receptor Trafficking Underlying a Form of Associative Learning. Science 308, 83-88 (2005).

21. Impey, S. et al. Stimulation of cAMP response element (CRE)-mediated transcription during contextual learning. Nature Neuroscience 1, 595-601 (1998).

22. Pi, H. J., Otmakhov, N., Lemelin, D., De Koninck, P. \& Lisman, J. Autonomous CaMKII Can Promote either Long-Term Potentiation or Long-Term Depression, Depending on the State of T305/T306 Phosphorylation. Journal of Neuroscience 30, 8704-8709 (2010).

23. Dillingham, B. C. et al. Fear Learning Induces Long-Lasting Changes in Gene Expression and Pathway Specific Presynaptic Growth. bioRxiv 571331 (2019) doi:10.1101/571331.

24. Sando III, R. et al. Inducible control of gene expression with destabilized Cre. Nat Meth 10, 1085-1088 (2013).

25. Tayler, K. K., Tanaka, K. Z., Reijmers, L. G. \& Wiltgen, B. J. Reactivation of Neural Ensembles during the Retrieval of Recent and Remote Memory. Current Biology 23, 99-106 (2013).

26. Tervo, D. G. R. et al. A Designer AAV Variant Permits Efficient Retrograde Access to Projection Neurons. Neuron 92, 372-382 (2016).

27. Xu, C. et al. Distinct Hippocampal Pathways Mediate Dissociable Roles of Context in Memory Retrieval. Cell 167, 961-972.e16 (2016).

28. LeGates, T. A. et al. Reward behaviour is regulated by the strength of hippocampus-nucleus accumbens synapses. Nature 564, 258-262 (2018).

29. Roy, D. S. et al. Memory retrieval by activating engram cells in mouse models of early Alzheimer's disease. Nature 531, 508-512 (2016).

30. Blair, H. T., Schafe, G. E., Bauer, E. P., Rodrigues, S. M. \& LeDoux, J. E. Synaptic Plasticity in the Lateral Amygdala: A Cellular Hypothesis of Fear Conditioning. Learn. Mem. 8, 229-242 (2001). 
31. Namburi, P., Al-Hasani, R., Calhoon, G. G., Bruchas, M. R. \& Tye, K. M. Architectural Representation of Valence in the Limbic System. Neuropsychopharmacology 41, 1697-1715 (2016).

32. Grewe, B. F. et al. Neural ensemble dynamics underlying a long-term associative memory. Nature 543, 670-675 (2017).

33. Otchy, T. M. et al. Acute off-target effects of neural circuit manipulations. Nature 528, 358-363 (2015).

34. Garner, A. R. et al. Generation of a Synthetic Memory Trace. Science 335, 1513-1516 (2012).

35. Tovote, P., Fadok, J. P. \& Lüthi, A. Neuronal circuits for fear and anxiety. Nat Rev Neurosci 16, 317-331 (2015).

36. Milad, M. R. \& Quirk, G. J. Fear Extinction as a Model for Translational Neuroscience: Ten Years of Progress. Annual Review of Psychology 63, 129-151 (2012).

37. Felsenberg, J. et al. Integration of Parallel Opposing Memories Underlies Memory Extinction. Cell 175, 709-722.e15 (2018).

38. Clem, R. L. \& Huganir, R. L. Calcium-Permeable AMPA Receptor Dynamics Mediate Fear Memory Erasure. Science 330, 1108-1112 (2010).

39. Clem, R. L. \& Schiller, D. New Learning and Unlearning: Strangers or Accomplices in Threat Memory Attenuation? Trends in Neurosciences 39, 340-351 (2016).

40. Shaban, H. et al. Generalization of amygdala LTP and conditioned fear in the absence of presynaptic inhibition. Nature Neuroscience 9, 1028-1035 (2006).

41. Humeau, Y., Shaban, H., Bissière, S. \& Lüthi, A. Presynaptic induction of heterosynaptic associative plasticity in the mammalian brain. Nature 426, 841-845 (2003).

42. Humeau, Y. et al. Dendritic Spine Heterogeneity Determines Afferent-Specific Hebbian Plasticity in the Amygdala. Neuron 45, 119-131 (2005).

Acknowledgments: We thank Anton Maximov for providing the Syn-Venus AAV, Thomas Hnasko for providing optogenetics equipment and Kathryn Fife for technical assistance, Ian Winchester and Yena Lee for assistance with the mouse colony and genotyping, and Natasha Weaver for administrative support during the years at The Scripps Research Institute. We are also thankful to Roberto Malinow, Stefan Leutgeb, Nicholas Spitzer, Thomas Hnasko and Kiriana Cowansage for helpful discussion and suggestions.

Funding: This work was supported by the National Institute of Health (grants R01MH057368 and R01DA035657, to M.M.) and by a Natural Sciences and Engineering Research Council of Canada discovery grant (\#418451-2013, to Q. Y.). L.M.C. was supported by the CAPES Foundation - Brazil (BEX 18818/12-9) and the Howard Hughes Medical Institute International Student Research Fellowship. A.F.S. was supported by the GABBA PhD program (FCT fellowship SFRH/BD/52037/2012).

Author contributions: L.M.C. and M.M. conceived the idea behind this work. L.M.C., Q.Y. and M.M. designed the experiments. L.M.C. and Q.Y. performed electrophysiological experiments. Q.Y. analyzed electrophysiological data. L.M.C. performed surgeries, behavior experiments, imaging and 
analysis. B.C.D. performed imaging analysis. A.F.S. and S.R. assisted with behavior experiments. W.D. assisted with tracing experiment. N.J. provided technical support. N.J. and M.M. designed and produced AAVs. E.J.Y. assisted with histological procedures. L.M.C. and M.M. wrote the manuscript. B.C.D., A.F.S., W.D. and Q.Y. edited the manuscript and contributed critically to data interpretation and discussion.

Competing interests: Authors declare no competing interests.

Data and materials availability: All data are available in the main text or the supplementary materials.

\section{Supplementary Materials:}

Materials and Methods

References: 43-51

Figures S1-S12

Tables S1-S2 